\title{
BMJ Open Incidence, diagnostic criteria and outcome following ventriculoperitoneal shunting of idiopathic normal pressure hydrocephalus in a memory clinic population: a prospective observational cross-sectional and cohort study
}

\author{
George Razay, ${ }^{1}$ Melissa Wimmer, ${ }^{2}$ lain Robertson ${ }^{3}$
}

To cite: Razay G, Wimmer M, Robertson I. Incidence, diagnostic criteria and outcome following ventriculoperitoneal shunting of idiopathic normal pressure hydrocephalus in a memory clinic population: a prospective observational cross-sectional and cohort study. BMJ Open 2019;9:e028103. doi:10.1136/ bmjopen-2018-028103

- Prepublication history and additional material for this paper are available online. To view these files, please visit the journal online (http://dx.doi org/10.1136/bmjopen-2018028103).

Received 28 November 2018 Revised 18 0ctober 2019 Accepted 24 October 2019

Check for updates

(C) Author(s) (or their employer(s)) 2019. Re-use permitted under CC BY-NC. No commercial re-use. See rights and permissions. Published by BMJ.

For numbered affiliations see end of article.

Correspondence to

Dr George Razay;

george.razay@ths.tas.gov.au

\section{ABSTRACT}

Objective To evaluate diagnostic criteria for idiopathic normal pressure hydrocephalus (INPH) among patients with memory impairment, and to estimate the incidence of INPH.

Design Prospective observational cross-section and cohort study of diagnostic accuracy.

Setting Memory Disorders Clinic following referral by the medical practitioners.

Participants 408 consecutive patients enrolled 20102014.

Outcome measures Reference diagnostic test was the clinical judgement of an experienced specialist based on the presence of cognitive impairment and/or balance and gait disorders in the presence of dilated ventricles. Mini-Mental State Examination (MMSE), Tinetti balance and gait tests were performed before and 12 months after ventriculoperitoneal shunt surgery. The association between reference diagnosis, clinical and brain CT scan measurements was estimated by multivariate Poisson regression. Triage index diagnostic test scores were calculated from the regression coefficients, with diagnostic thresholds selected using receiver operating characteristic analysis.

Results The presence of balance and/or gait disorders, especially fear of falling, difficulty standing on toes/ heals, urinary disturbances, ventriculomegaly with Evans ratio greater than Combined Diagnostic Threshold (0.377-\{Maximum width of posterior horns $\left.{ }^{\star} 0.0054\right\}$ ), strongly predict the diagnosis of INPH; while hallucinations and/or delusions and forgetfulness reduce the likelihood of the diagnosis. This triage index test had high sensitivity (95.2\%) and specificity (91.7\%). 62 of 408 (15\%) participants with cognitive impairment had INPH, an incidence of 11.9/100 000/year and 120/100 000/year over 75 years. $96 \%$ of participants following shunting, compared with $45 \%$ of the non-shunted, improved by over $25 \%$ of available measurable improvement in either MMSE or balance/gait scores ( $51 \%$ difference; $95 \% \mathrm{Cl} 28 \%$ to $74 \% ; p<0.001$ ), and $56 \%$ vs $5 \%$ improved by over $50 \%$ of maximum in both (51\% difference; $95 \% \mathrm{Cl} 30 \%$ to $73 \%$; $p<0.001)$.
Strengths and limitations of this study

- This study has developed a triage index test that might be applied by non-specialist practitioners to select patients with a preliminary the diagnosis of idiopathic normal pressure hydrocephalus (INPH) who might be suitable for specialist INPH management.

- This is a prospective study within a define population and well-established clinical service with detailed clinical and radiological assessments that is likely to identify the majority of patients with INPH.

- Internal validation supported the triage index test values.

This preliminary study requires external validation in different and larger populations.

Conclusion The triage index test score is a simple tool that may be useful for physicians to identify INPH diagnoses and need for referral for shunt surgery, which may improve cognitive, balance and gait functioning.

\section{INTRODUCTION}

Idiopathic normal pressure hydrocephalus (INPH) is one of the few potentially treatable causes of dementia. Diagnosis is based on the clinical triad of gait disturbance, cognitive decline and urinary incontinence in the presence of enlarged ventricles. The condition is treated by diversion of cerebrospinal fluid, most commonly by the insertion of a ventriculoperitoneal (V-P) shunt. ${ }^{1-4}$ INPH generally occurs in older people ${ }^{56}$ However, diagnosis is difficult since the symptoms and ventriculomegaly could be attributed to other neurodegenerative diseases. ${ }^{4}$ Moreover, no definitive method exists to confirm the diagnosis, and the only validation of the diagnosis is improvement following shunt surgery. Guidelines for INPH diagnosis have 
been recommended including Evans ratio $>0.3^{3}$ and the disproportionately enlarged subarachnoid space hydrocephalus (DESH) criteria, ${ }^{7}$ but these guidelines are mainly used in tertiary referral centres, and it is left to the skills of experienced specialists to make the diagnosis and recommend treatment. However, there is still wide variation in the diagnostic criteria, which may lead to misdiagnosis as other forms of dementia. ${ }^{8}$ Thus, there have been few reliable estimates of the incidence of INPH, which has led to it being viewed as a rare condition. ${ }^{489}$ There is also a need for a triage screening test that is simple and accurate enough to be used by the general practitioners in routine practice.

The aims of this study are to: (1) estimate the incidence of INPH among patients with memory impairment; (2) describe the diagnostic features of patients diagnosed with INPH using the Launceston reference criteria, by comparing the clinical and radiological features of INPH with non-INPH patients; (3) create triage index tests before and after brain CT scan; (4) estimate their diagnostic accuracy against the reference (specialist) diagnosis and (5) test the validity of the reference diagnostic criteria by estimating the change in cognitive, balance and gait functioning following V-P shunting.

\section{METHODS}

\section{Study design and setting}

This was a prospective observational cross-sectional and cohort study. Consecutive patients with memory impairment referred by general practitioners between April 2010 and July 2013 to the Launceston Memory Disorders Clinic, the only such clinic in Northern Tasmania, were recruited. A Standards for Reporting Diagnostic accuracy studies (STARD) flow diagram of participant numbers for the overall study is shown in online supplementary materials, figure $1 \mathrm{~S}$.

\section{Patient and public involvement statement}

While there was no formal involvement of patients or their representatives in the planning, design and performance of this research, prior to this study, the specialist (GR) had been contacted by the patients and their relatives from other Australian states with memory problems seeking consultations and treatments following publicity arising from previous research publication. Those patients had been unable to access suitable specialists willing and able to undertake the appropriate investigations locally. Part of the motivation for this research was to assist those services to become familiar and comfortable with delivering the appropriate investigations and treatments desired by patients. Regular meetings with the participants were held to inform them of the progress and results of this study.

\section{Data collection}

All participants underwent standard clinic assessment, and the reference diagnosis was made by the same specialist physician/geriatrician (GR) ${ }^{10}$ Data included detailed history of memory, balance, gait and urinary symptoms including features suggesting dementia such as aphasia, agnosia, apraxia, hallucination, delusion, agitation, aggressive behaviour and change in personality. Examination included cognitive function using the MiniMental State Examination (MMSE), ${ }^{11}$ and balance and gait functions by standing with eyes closed and on toes and heels, and the tandem walk test (walking $2 \mathrm{~m}$ heel to toe). All patients had a brain CT scan, and an additional MRI for suspected INPH patients.

The diagnosis of non-INPH dementia was made according to the Diagnostic and Statistical Manual of Mental Disorders, fourth edition, and the diagnosis of Alzheimer's disease (AD), vascular and mixed dementia, frontal lobe dementia, Lewy body dementia, and Parkinson's disease dementia, were made according to standard criteria. ${ }^{12}$ Mild cognitive impairment was diagnosed using the Petersen criteria. ${ }^{13}$

\section{Definition of diagnostic reference standard}

INPH was diagnosed by the specialist physician (GR) using his routine practice. This was based on fulfilling three criteria: (1) cognitive impairment (not necessarily dementia), and balance and gait disorder; (2) dilated ventricles, (regardless of cerebral atrophy and white matter ischaemia) and (3) no evidence of an antecedent event such as head trauma, meningitis, intracerebral haemorrhage or other medical conditions sufficient to explain the presenting symptoms. Symptoms and signs of Parkinsonism and absence of balance/gait problems reduced the suspicion of INPH and reduce the likelihood for V-P shunting.

In addition to the routine clinical practice observations, additional historical, symptomatic, examination and radiological observations were made by the specialist and study coordinator (as described in study protocol in online supplementary materials). These observations were used after the end of the study to calculate the triage index test scores: the specialist was aware of some of these observations at the time he made the reference standard diagnosis but was not aware of the final index test scores.

To validate INPH diagnoses, the response to V-P shunting was measured. Participants with INPH underwent assessment by an independent observer at baseline, and followed up at 3, 6 and 12 months. The primary efficacy measure was the Clinician's Interview Based Impression of Change Plus Caregiver Interview (CIBIC-plus) ${ }^{14}$ : evaluating global, cognitive, balance, gait and urinary functioning with 7-point scale $(+3$, markedly improved relative to baseline; -3 markedly worse). Secondary efficacy measures included the MMSE for the assessment of cognition, and Tinetti balance and gait tests ${ }^{15}$ for assessment of mobility.

Participants were informed about the study at the time of diagnosis. The intention was to treat all INPH participants with a V-P shunt. Participants who agreed to consider surgery were referred to a single neurosurgical 
department in Hobart, southern Tasmania, which made the final decisions about shunt surgery. Patients who did not have surgery were used as comparators.

\section{Definition of index test}

Two simplified screening tools for potential use by nonspecialised practitioners (triage tests) to select participants for brain CT scan and then referral to specialists for further evaluation and treatment were constructed by estimating the association between the presence of INPH and clinical and radiological findings using multivariate regression analysis. Brain CT scan of 60 participants with non-INPH were randomly selected using the randomnumber generator function in Microsoft Excel. The detailed CT scan measurements were performed after completion of the study for comparison with the reference standard and were not used as criteria for the initial diagnosis and indication for referral for shunt surgery. Selection of the variables to be included in the index test predictor models was driven by the automated processes of the stepwise regression facility in the Stata statistical package, and before the diagnostic accuracy tests for the index tests were applied.

\section{Sample size}

Sixty participants with INPH and 400 participants overall were required to detect a mean 3-point improvement in MMSE score in the shunted participants compared with zero change in those not shunted (assuming an SD of change of 4 ; alpha 0.05 ; power $80 \%$; $50 \%$ patients shunted).

\section{Statistical analysis}

The incidence of new diagnoses of INPH in the Memory Clinic population was estimated in the Northern Tasmania population (Australian Bureau of Statistics, Census 2011), with direct age-gender standardisation to the equivalent Australian population.

The clinical and radiological observations recorded at initial diagnosis in participants with and without an INPH were compared in order to construct a triage index test for INPH diagnosis. A multivariate Poisson regression model was used to estimate the association between the INPH diagnosis and an optimum combination of clinical and CT scan features. The predictor variables were selected using backward stepwise regression from a list included in table $1 \mathrm{~S}$ of online supplementary materials table 1S. From the CT scan, several measurements were obtained including Evans ratio (the maximum bifrontal distance divided by the maximum inner diameter of the skull at the same level in transverse views of the brain) (figure 1), ${ }^{16}$ maximum width of anterior and posterior horns; cerebral atrophy and periventricular white matter ischaemia. ${ }^{17}$ The association between Evans ratio and maximum width of posterior horns (MWPH) was examined graphically, and a method was identified for a patientspecific modified Evans ratio threshold (Combined Diagnostic Threshold, CDT). A total of $233(3.0 \%)$

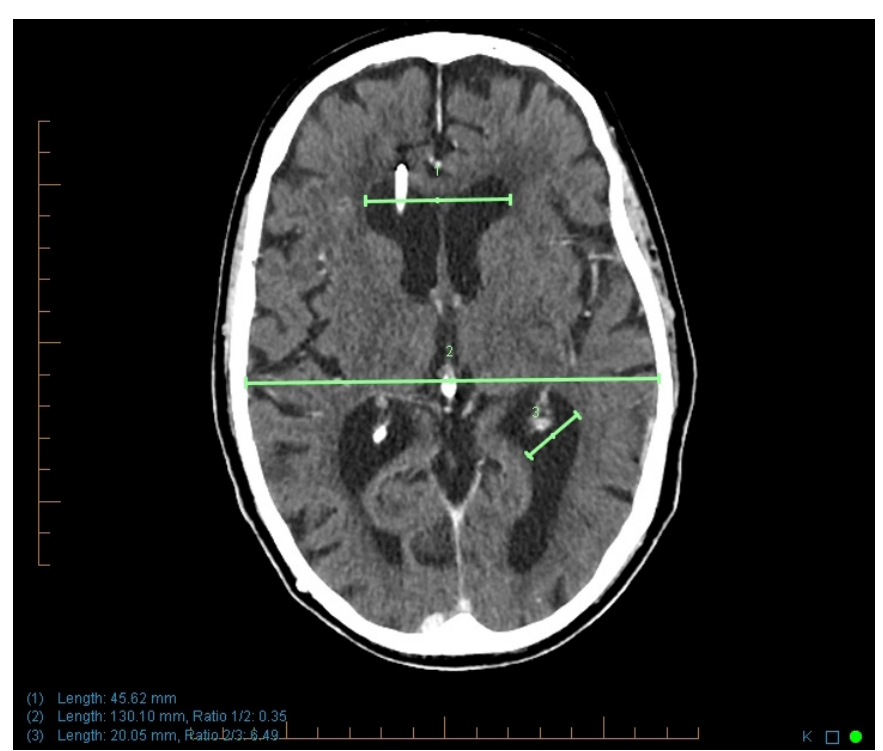

Figure 1 Example of axial brain CT scan showing Evans ratio (the maximum bifrontal distance) (1) divided by the maximum inner diameter of the skull (2) and the maximum width of the posterior horn (3).

missing observations occurred in a total of 7752 required cognitive and urinary observations. No missing observations occurred in the balance/gait and CT scan domains. A total of $377(92.4 \%)$ of 408 patients had complete data recorded. Missing data were substituted by multiple imputation using the variables in online supplementary materials table 1S. The regression coefficients from the optimum Poisson regression models were then used to calculated risk scores for each patient based on a linear additive combination of variables. Two risk scores were calculated from the Poisson regression analysis before and after CT scan results. The risk scores were examined using receiver operating characteristic (ROC) analysis to identify a threshold (the index test) with approximately 95\% sensitivity, with estimation of ROC specificity and area for those tests. (See online supplementary appendix 1: Additional Description of Statistical Methodology in online supplementary materials for detailed description of the study analysis, including internal validation of the proposed risk scores using multiple bootstrap sampling. This appendix includes online supplementary tables 2S-9S showing intermediate supporting results of the analysis).

The association between the Evans ratio and the cerebral atrophy score was estimated using ordered logistic regression. The compliance of each INPH patient with the International Guideline criteria was determined in four domains (cognitive, balance/gait, urinary, imaging), and the agreement between the International Guidelines diagnosis and the reference diagnostic criteria was tested by the inter-rater agreement kappa statistic. The change in cognitive, balance/gait, and CIBIC-plus scores were compared in participants with or without V-P shunt in the first year following diagnosis, using repeated measures 
Table 1 Diagnoses and demographic characteristics of study participants

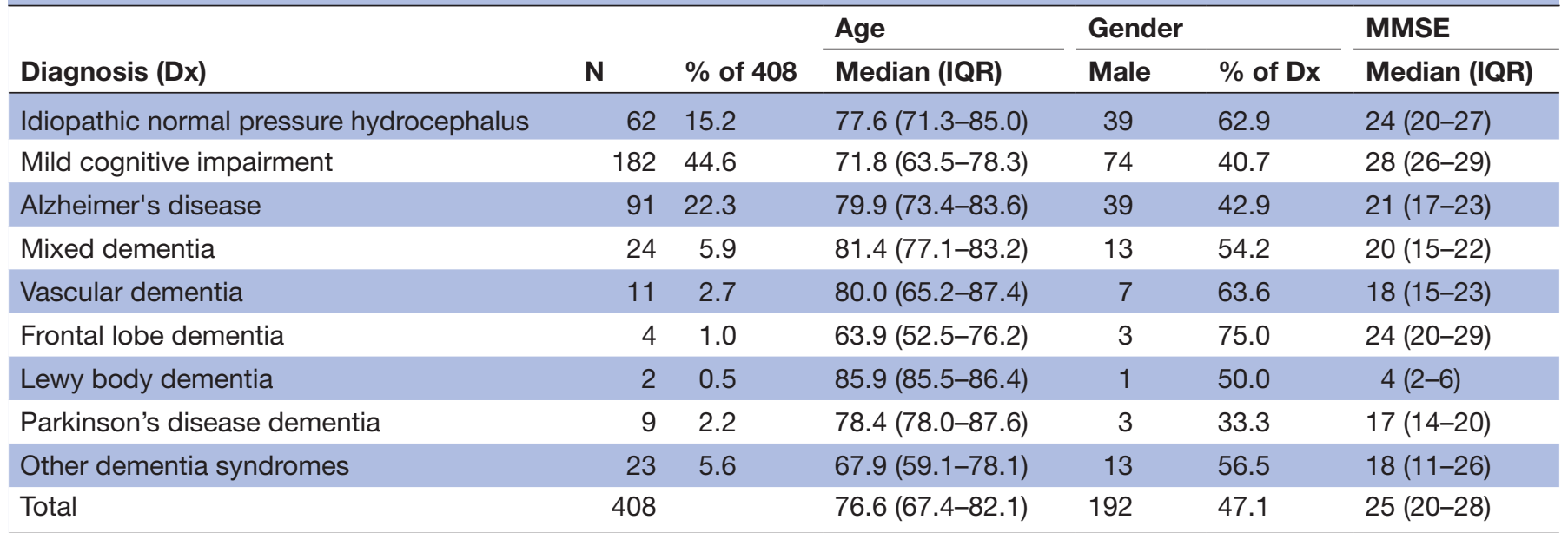

MMSE, Mini-Mental State Examination.

mixed effects linear regression, adjusted for initial scores at diagnosis.

The protocol for this study is included at the end of the online supplementary material.

All analyses were performed using Stata/MP2 V.14.2 (StataCorp).

\section{RESULTS}

\section{Baseline characteristics and diagnoses of patients}

A total of 408 consecutive participants with memory impairment were enrolled, of whom 215 (53\%) were women. Their median age was 76.6 years (range $32.6-94.8$ years). Table 1 shows the diagnoses and demographic characteristics of the participants. Median duration of symptoms was 24 months (IQR 12-42 for INPH; 18-54 for non-INPH participants). Sixty-two (15.2\%; 95\% CIs $11.9 \%$ to $19.1 \%$ ) participants had INPH. The flow diagram of the participants is shown in online supplementary materials figure $1 \mathrm{~S}$.

\section{The incidence of INPH}

A total of 376 participants were from Northern Tasmania (2011 census population of 137560 ), 29 were from Northwestern Tasmania, and 3 were from Southern Tasmania. Fifty-four Northern Tasmanian INPH participants over 3.3 years (time between first and last recruitment) represent a minimum incidence of new diagnosis of INPH of at least $11.9 / 100000 /$ year (95\% CI 9.0 to 14.8 ; age-sex standardised 10.1; 95\% CI 7.4 to 13.5 ) (online supplementary table 10S). The raw incidence increased with age, with the highest incidence of 120/100 000/year in those 75-95 years old.

\section{Clinical characteristics of participants}

The frequency of different complaints, signs and CT scan results is shown in online supplementary table $1 \mathrm{~S}$. For individual features, there was considerable overlap between INPH and non-INPH participants, and a high degree of cross-correlation between the presence of different variables. Fifty-nine of 62 participants with an initial diagnosis of INPH had cognitive and balance deficits, with ventriculomegaly on CT scan. Five of the 60 randomly selected participants with an initial diagnosis of non-INPH had cognitive and balance deficits with ventriculomegaly, three of whom had Parkinsonism and two had $\mathrm{AD}$ combined with INPH. These participants were not considered for V-P shunting. Moreover, six participants had ventriculomegaly, but were not considered to have INPH by both the reference and index test criteria. The reasons for accepting or rejecting a diagnosis of INPH in 62 INPH participants and a randomly selected sample of 60 of 346 non-INPH participants are shown in online supplementary table $11 \mathrm{~S}$.

Two index tests were constructed to reproduce the diagnosis of INPH made by the specialist, before and after a CT scan, based on multivariate Poisson regression analysis. Prior to the CT scan, the presence of balance/gait problems, failure of both stand-on-toes and stand-on-heels tests, a fear of falling, presence of urinary disturbance (all of urinary urgency, nocturnal frequency and incontinence) were the main predictors of INPH (table 2). Being more forgetful (Forgetful score $\geq 3$; =immediate recall score on MMSE $<2$ (scoring 1=1, 0=2)+forgets names+forgets appointments+forgets recent conversations), and the presence of delusions/hallucinations were mildly negatively associated with INPH. When the individual predictors were combined into a risk score, the overlap between the INPH and non-INPH patients was reduced compared with any individual variable. ROC analysis identified a threshold for the risk score of -2.25 , which identified 59 of 62 cases of INPH (sensitivity 95.2\%), while excluding 221 of 346 non-INPH cases (specificity of $64.7 \%$ ). Internal validation of the risk scores demonstrated that there was only a minor 'optimism' bias in the risk score prior to CT scans generated directly from the study data, compared with that generated from the coefficients derived from 
Table 2 Association between the presence of idiopathic normal pressure hydrocephalus (INPH) and its clinical predictors before CT scans

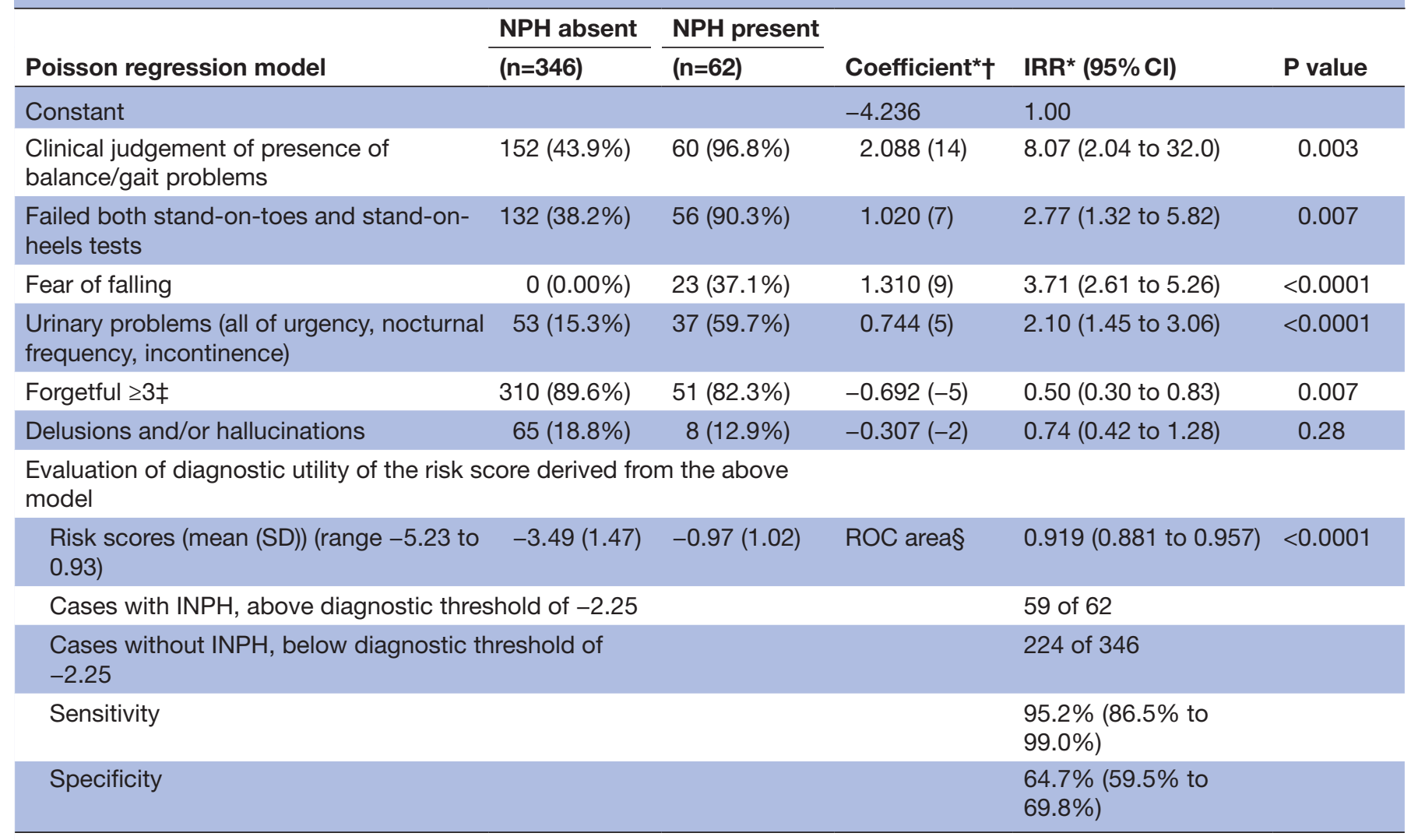

*Association between diagnosis of INPH and predictor variables, estimated using stepwise multivariate Poisson regression (IRR; $95 \%$ Cls; $p$ value) with included variables selected from a list including (on history or examination): balance/gait problems, all urinary problems present (urgency, frequency, incontinence), age, gender, MMSE, agnosia, apraxia, failed gait/balance tests (1) stand-eyes-closed, (2) stand-ontoes and stand-on-heels, (3) walk 2 m straight, (4) brisk reflexes present, (5) rigidity, (6) bradykinesia, (7) fear-of-falling, agitation, delusions, hallucinations, reading problems, expressive aphasia, personality change, forgets recent conversations, appointments, or names, MMSE immediate recall $<2$. Missing data were substituted by multiple imputation.

†Risk scores were calculated from the regression coefficients using equation 1; either the raw coefficient, or an integer rounding of (coefficient/0.15) (in brackets). The sensitivity and specificity performance of the raw coefficient risk score and the integer rounding risk score was identical.

łForgetful: presence of 3 or 4 of (1) immediate recall score on MMSE <2; (2) forgets names; (3) forgets appointments and (4) forgets recent conversations.

$\S R O C$ analysis was used to evaluate and select diagnostic thresholds for the risk scores.

IRR, incidence rate ratio; MMSE, Mini-Mental State Examination; ROC, receiver operating characteristic.

the bootstrap sampling validation process (specificity of $62.1 \%)$. No differences were seen following the CT scans in the specificity $(91.7 \%)$ or the identity of the patients chosen between the initial and validation risk scores.

Evans ratio and MWPH were most strongly associated with the reference diagnosis of INPH. Figure 2 shows the relationship between CT scan Evans ratio and MWPH. Ventriculomegaly, defined as an Evans ratio above a patient-specific CDT (with a formula (0.377$\left.\left\{\mathrm{MWPH}^{*} 0.0054\right\}\right)$ ), was found by itself to identify 60 of 62 INPH participants (sensitivity 96.7, 95\% CI 88.7 to $99.6 \%)$ and excluded 49 of 60 non-INPH participants (specificity 81.7 (95\% CI 69.6 to $90.5 \%$ ). The example CT scan shown in figure 1 has an Evans ratio of 0.35 and MWPH of $20.1 \mathrm{~mm}$ : thus, the patient has a CDT of 0.268 $(0.377-\{20.1 * 0.0054\})$, and the Evans ratio is well above that threshold. In this study, the CDT appeared superior to alternative definitions of ventriculomegaly: a threshold for Evans ratio $\geq 0.275$ with $96.7 \%$ sensitivity had a specificity of $76.7 \%$; a threshold of $\geq 0.30$ had a sensitivity of $77.4 \%$ and a specificity of $85.0 \%$.

Moreover, cerebral atrophy grade was associated with increasing Evans ratio in non-INPH participants (OR $2.05 ; 95 \%$ CI 1.20 to $3.53 ; \mathrm{p}=0.009)$, but not in INPH participants (OR 0.83 ; $95 \%$ CI 0.51 to $1.35 ; \mathrm{p}=0.46$ ).

When the results of the brain CT scan were included in the multivariate analysis (table 3), the diagnosis of INPH was predicted by the presence of balance and/or gait problems, fear of falling, urinary disturbance and ventriculomegaly (Evans ratio >CDT); while hallucinations and/ or delusions were negatively predictive. Individual variables were not strongly predictive, but a threshold value of -1.0 for the post-CT index test showed a sensitivity of $95.2 \%$ ( $95 \%$ CI $86.5 \%$ to $99.0 \%$ ), and a specificity of $91.7 \%$ 


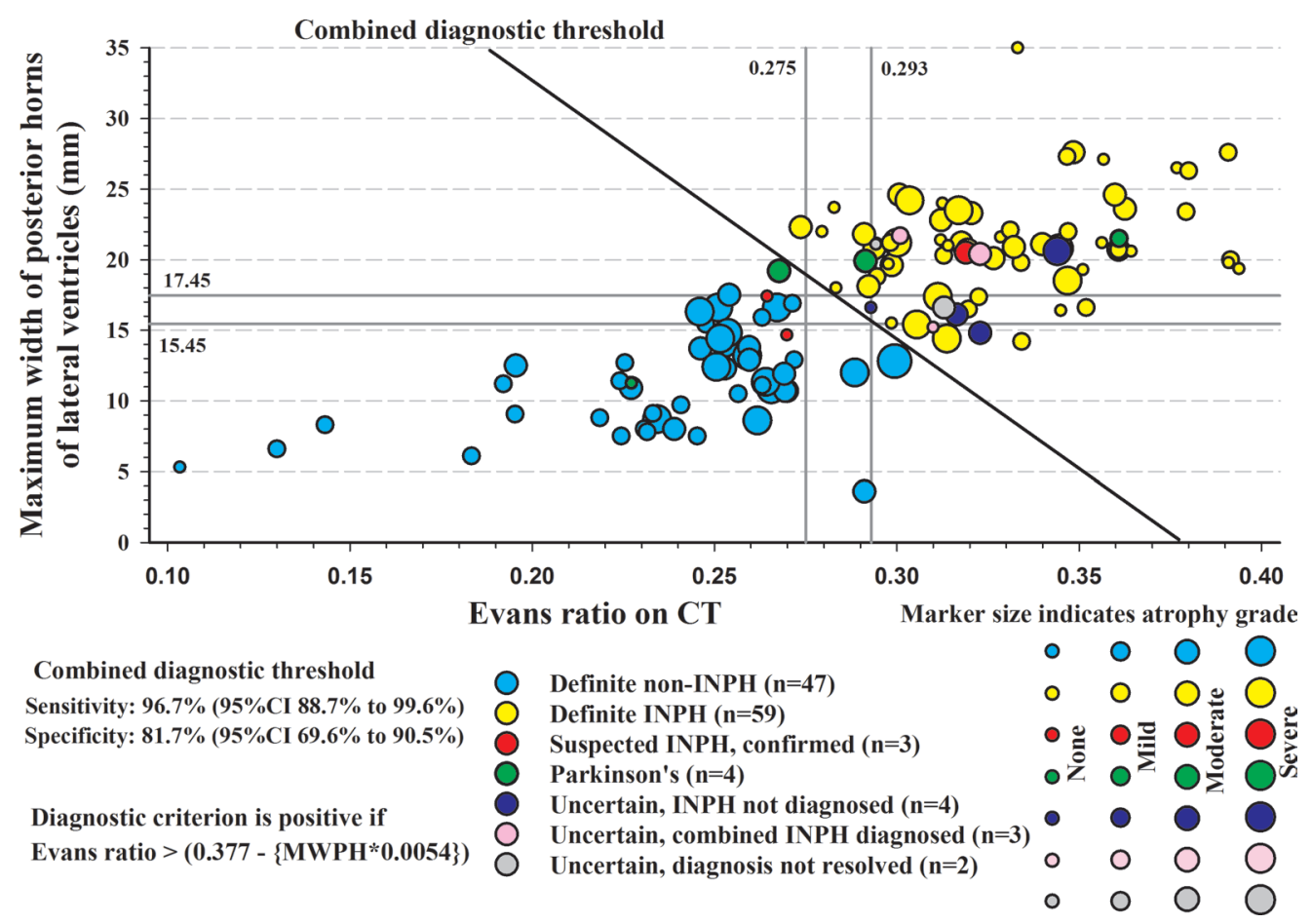

Figure 2 Relationship between Evans ratio and the maximum width of the posterior horn of the lateral ventricles on CT scan in 122 study participants diagnosed as INPH or non-INPH at initial assessment. INPH, idiopathic normal pressure hydrocephalus; $\mathrm{MWPH}$, maximum width of posterior horns.

(95\% CI $81.6 \%$ to $97.2 \%$ ). If the patients with combined INPH and Alzheimer's or Parkinsonism were counted as INPH, sensitivity $(95.5 \%)$ and specificity $(98.2 \%)$ of the index test were increased.

Simplified score sheets (figure 3) for predicting a diagnosis of INPH were developed from coefficients of the Poisson regression models in tables 2 and 3 (by rounding of (coefficient/0.15)). Participants with memory problems and a total simplified score $\geq 14$ require a brain CT scan (sensitivity $95.2,95 \%$ CI 86.3 to $99.0 \%$ and specificity $63.3 \%, 95 \%$ CI $58.0 \%$ to $68.4 \%$ ). After measurements of Evans ratio and MWPH, a total score $\geq 18$ suggested the need for referral to neurosurgeons for consideration of V-P shunting. The simplified risk scores before and after CT scan had an identical performance to the precise risk scores, including and excluding the same participants (sensitivity 95.2 and specificity $91.7,95 \%$ CI 81.6 to $97.2 \%)$. The value of the individual participant scores is shown in online supplementary table 12S. Comparison of the clinical and CT scan components of the reference test criteria and International Guidelines is shown in online supplementary tables 13S, 14S. There was agreement in $98.4 \%$ of cognitive, $87.1 \%$ of balance and gait, $95.2 \%$ of urinary and $74.2 \%$ of CT scan assessments. In 54 (87.1\%) of the patients, there was an agreement between the reference test criteria and International Guidelines for the diagnosis of probable INPH, and seven patients fulfilled the reference test criteria and International Guidelines for the diagnosis of possible INPH.

Follow-up of participants over 12 months occurred to validate the diagnoses of INPH. All were considered for V-P shunting, of whom 29 (47\%) had a shunt and 33 participants were not shunted (table 4 shows the reason for not shunting). Follow-up of participants was less complete in the non-shunted group due to difficulty and unwillingness to attend and comply with assessments. Thus, 25 shunted and 21 non-shunted participants were examined at initial assessments, and at either 6 and/or 12-month visits.

Participants who were shunted, compared with the non-shunted, had significantly higher initial MMSE scores (25.7 vs 19.8; mean difference $6.0 ; 95 \%$ CI 3.2 to 8.7; $<<0.001)$, and similar initial Tinetti balance and gait scores ( 14.5 vs 13.9 ; mean difference $0.6 ; 95 \% \mathrm{CI}-2.3$ to 3.5; $\mathrm{p}=0.68$ ). In the shunted group, the mean MMSE score improved significantly by about $72 \%$ of the maximum available improvement (4.75 at 12 months of a possible 6.1 (ie, 30-23.9)), with the greatest improvement being at 12 months. The mean Tinetti scores improved by about $85 \%$ for balance and gait scores $(90 \%$ for balance and $78 \%$ for gait) with maximum improvement at 6 months (table 5). Moreover, subjective assessments using the CIBIC-plus rating indicated that participants following shunting were very much improved on global, cognitive, balance and gait and moderately improved on urinary functioning (table 6). There was no association between the proportional change in cognitive (OR 0.96; 95\% CI 0.60 to 1.52; $\mathrm{p}=0.87$ ), balance and gait function (OR 0.93; $95 \%$ CI 0.32 to $2.68 ; \mathrm{p}=0.90$ ) and the index test scores derived from the clinical and CT scan assessments.

The proportional change in MMSE and Tinetti balance/ gait scores in the shunted and non-shunted participants at 
Table 3 Association between the presence of idiopathic normal pressure hydrocephalus (INPH) and its clinical and CT scan predictors

\begin{tabular}{|c|c|c|c|c|c|}
\hline \multirow[b]{2}{*}{ Poisson regression model } & \multirow{2}{*}{$\begin{array}{l}\text { NPH absent } \\
(n=60)^{*}\end{array}$} & \multirow{2}{*}{$\frac{\text { NPH present }}{(n=62)}$} & \multirow[b]{2}{*}{ Coefficient } & \multirow[b]{2}{*}{ IRR† (95\% Cl) } & \multirow[b]{2}{*}{$P$ value } \\
\hline & & & & & \\
\hline Constant & & & -3.798 & & \\
\hline $\begin{array}{l}\text { Clinical judgement of presence of balance/ } \\
\text { gait problems }\end{array}$ & $25(41.7 \%)$ & $60(96.7 \%)$ & $1.267(8)$ & 3.55 (0.93 to 13.6$)$ & 0.064 \\
\hline $\begin{array}{l}\text { Urinary problems (all of urgency, nocturnal } \\
\text { frequency, incontinence) }\end{array}$ & $7(11.7 \%)$ & $37(59.7 \%)$ & $0.199(1)$ & $1.22(1.02$ to 1.45$)$ & 0.014 \\
\hline Forgetful $\geq 3 \ddagger$ & $53(88.3 \%)$ & $51(82.3 \%)$ & $-0.203(-1)$ & $0.82(0.64$ to 1.04$)$ & 0.11 \\
\hline Delusions and/or hallucinations & $12(20.0 \%)$ & $8(12.9 \%)$ & $-0.136(-1)$ & 0.87 (0.68 to 1.13$)$ & 0.30 \\
\hline Cases with NPH above diagnostic thresholc & d of -1.0 & & & 59 of 62 & \\
\hline Cases without NPH below diagnostic thresh & cold of -1.0 & & & 55 of 60 & \\
\hline Sensitivity & & & & $\begin{array}{l}95.2 \%(86.5 \% \text { to } \\
99.0 \%)\end{array}$ & \\
\hline Specificity & & & & $\begin{array}{l}91.7 \%(81.6 \% \text { to } \\
97.2 \%)\end{array}$ & \\
\hline
\end{tabular}

${ }^{*}$ A sample of 60 participants was selected randomly from the 346 non-INPH participants.

$\dagger$ The variables for inclusion in the multivariate model were selected by backward stepwise Poisson regression from a list including (on history or examination) balance/gait problems, all urinary problems present (urgency, frequency, incontinence), age, gender, MMSE, agnosia, apraxia, failed gait/balance tests 1 (stand-eyes-closed), 2 and 3 (stand-on-toes and stand-on-heels), 4 (walk $2 \mathrm{~m}$ straight), 5 (brisk reflexes present), 6 (rigidity), 7 (bradykinesia), 8 (fear-of-falling), agitation, delusions, reading problems, expressive aphasia, personality change, forgets recent conversations, appointments, or names, immediate recall $<2$, hallucinations, (on CT scan) severe atrophy, severe leucoaraiosis, enlarged temporal horns, space-occupying lesion, dilated Silvian fissure, temporal lobe thickening, ventriculomegaly defined as Evans ratio $>$ (3.77$\left\{\mathrm{MWPH}^{*} 0 \cdot 0054\right\}$ ), width between caudate nuclei at the foramen of Munro, maximum width of third ventricle, and minimum width of both cella media, maximum width of anterior horns, MWPH. Missing data were substituted by multiple imputation.

¥Risk scores were calculated from the regression coefficients using equation 1; either the raw coefficient, or an integer rounding of coefficient/0.15 (in brackets). The sensitivity and specificity performance of the raw coefficient risk score and the integer rounding risk score was identical.

IRR, incidence rate ratio; MMSE, Mini-Mental State Examination; MWPH, maximum width of posterior horns; ROC, receiver operating characteristic.

follow-up visits is shown in figure 4 . The majority of participants with available follow-up data who were shunted (24 of $25(96 \%)$; vs 9 of 21 non-shunted patients (45\%)) improved by at least $25 \%$ of the available measurable improvement in either MMSE or balance/gait scores compared with the non-shunted participants (improvement difference $51 \%$; $95 \%$ CI $28 \%$ to $74 \%$; p $<0.001$ ), and the majority of participants improved by at least $50 \%$ of maximum in both MMSE and balance/gait scores (14 of $25(56 \%)$ shunted vs 1 of 21 (5\%) non-shunted; improvement difference $51 \%$; $95 \%$ CI $30 \%$ to $73 \%$; p $<0.001)$. The Launceston reference criteria and International Guidelines criteria showed similar response to shunting, although only three of the participants who were positive for only the Launceston criteria were shunted.

\section{DISCUSSION \\ Key findings}

This study demonstrates a triage index test that predicts the diagnosis and incidence of INPH among patients with memory impairment. The presence of balance and/or gait disorders with difficulty standing on toes and heals, fear of falling; urinary urgency, nocturnal frequency and incontinence; ventriculomegaly with Evans ratio above CDT (0.377\{MWPH*0.0054\}), strongly predict the diagnosis of INPH, while hallucinations and/or delusions; and forgetfulness, reduce the likelihood of the diagnosis (seven-item check list, figure 3). The study also confirms that V-P shunting is associated with improvements in cognitive, balance, gait and urinary functioning of patients with INPH in the first year; there was no indication of lesser improvements in patients with less intensity of symptoms.

The study also suggests INPH is more common in our population than has been previously reported. At least 


\begin{tabular}{|c|c|c|c|c|c|c|c|}
\hline & & \multicolumn{3}{|c|}{ Before CT scan } & \multicolumn{3}{|c|}{ After CT scan } \\
\hline & Observation & Present & $\begin{array}{l}\text { Score } \\
\text { value }\end{array}$ & Score & Present & $\begin{array}{c}\text { Score } \\
\text { value }\end{array}$ & Score \\
\hline 1 & Complaint of balance problems & & 14 & & & 8 & \\
\hline 2 & Fails both standing on heals and toes tests & & 7 & & & 0 & \\
\hline 3 & Fear of falling during usual activities & & 9 & & & 1 & \\
\hline 4 & $\begin{array}{l}\text { Significant forgetfullness } \\
\text { (1) Forgets names of people }(\text { score }=1) \\
\text { (2) Forgets appointments }(\text { score }=1) \\
\text { (3) Forgets conversations }(\text { score }=1) \\
\text { (4) Scores } 0 \text { (score }=2 \text { ) or } 1(\operatorname{score}=1) \\
\text { for immediate Recall components } \\
\text { of MMSE } \\
\text { Score of } 3-5 \text { total for these tests }\end{array}$ & & -5 & & & -1 & \\
\hline 5 & Hallucinations or delusions & & -2 & & & -1 & \\
\hline 6 & $\begin{array}{l}\text { Presence of all of urinary incontinence, } \\
\text { urgency, and nocturnal frequency }\end{array}$ & & 5 & & & 1 & \\
\hline 7 & $\begin{array}{l}\text { Ventriculomegaly: } \\
\text { Evans ratio }>(0 \cdot 377-\{\text { MWPH*0.0054\}) }\end{array}$ & & & & & 16 & \\
\hline Total & \multicolumn{3}{|c|}{$\begin{array}{l}\text { A total score before CT scan of } 14 \text { points or more suggests } \\
\text { the need for CT scan }\end{array}$} & & & & \\
\hline Total & \multicolumn{6}{|c|}{ A total score after $\mathrm{CT}$ scan of 18 points or more suggests need for neurosurgical referral } & \\
\hline
\end{tabular}

Figure 3 Clinical scoring sheet: a simplified risk scoring system to be applied in patients presenting with cognitive problems to determine whether a brain CT scan and subsequently a referral to a neurosurgeon are necessary. MMSE, Mini-Mental State Examination; MWPH, maximum width of posterior horns.

$15 \%$ of patients seen in our memory disorders clinic may have INPH.

\section{Comparison with other studies}

The incidence of INPH

Studies of INPH report an incidence of 1.2-5.5/100 000/ year, $^{4-6} 18$ with shunt surgery about $1 / 100000 /$ year. $^{4} 19$ INPH is stated to be a rare cause of dementia. ${ }^{8}$ A metaanalysis of 39 studies between 1987 and 2001 reported INPH in $1 \%$ of dementia patients. ${ }^{8}$ We found INPH more common, with an incidence of 11.9/100 000/year with

\begin{tabular}{llr}
\hline Table 4 & Reasons for not performing V-P shunting & \\
\hline Reasons & & 9 \\
\hline & Medically unfit for surgery & 12 \\
& Patient or family's refusal & 4 \\
& $\begin{array}{l}\text { Intervening comorbidities (metastatic } \\
\text { cancer n=2, stroke n=1, bowel } \\
\text { infarction n=1) }\end{array}$ & \\
& Presence of parkinsonism & 2 \\
& $\begin{array}{l}\text { Achondroplasia } \\
\text { No available consent }\end{array}$ & 1 \\
& $\begin{array}{l}\text { Waiting for symptoms to deteriorate } \\
\text { sufficiently }\end{array}$ & 1 \\
& Left Tasmania or lost to follow-up & 2 \\
& Withdrawn from study & 1 \\
\hline
\end{tabular}

V-P, ventriculoperitoneal. the rate increasing with age. The true incidence of INPH is probably higher as some patients may not have cognitive impairment, ${ }^{620}$ or asymptomatic with ventricular enlargement at the time of assessment. ${ }^{20} 21$

The differences in the reported incidence of INPH may be due to selection and ascertainment biases, and retrospectivity causing non-detection of some less florid cases; having different age groups; and using inconsistence diagnostic and inclusion criteria. Thus, INPH is probably misdiagnosed, underdiagnosed and undertreated. ${ }^{19}{ }^{22}$ However, it is possible that there are true variations in incidence rates in different populations.

\section{The diagnostic criteria}

There is no consensus on common criteria to diagnose INPH and predict beneficial response to shunt surgery. Various scales and guidelines have been recommended to facilitate a more accurate diagnosis, and to select appropriate patients who might benefit from shunt surgery. ${ }^{3723}$ They are still not widely used: of 15 studies conducted following publication of the guidelines, only six followed the recommended guidelines, ${ }^{20}$ possibly due to their lack of clarity about how to combine the different symptoms, signs and brain imaging. Thus, the skill and experience of a specialist remains the reference test for INPH diagnosis.

Our triage index test score, a short seven-item check list (figure 3), could be used to select patients with cognitive impairment with a presumptive diagnosis of INPH for neurosurgical evaluation and treatment. The criteria were efficient at discriminating INPH from non-INPH patients 
Table 5 Change in objective cognitive and balance/gait scores over the 12 months following initial assessment in shunted and non-shunted participants

\begin{tabular}{|c|c|c|c|c|c|c|c|c|}
\hline & \multicolumn{3}{|c|}{ Non-shunted } & \multicolumn{3}{|c|}{ Shunted } & \multicolumn{2}{|c|}{$\begin{array}{l}\text { Comparison: shunted versus } \\
\text { non-shunted patients }\end{array}$} \\
\hline & $\mathbf{N}$ & Mean & SD & $\mathbf{N}$ & Mean & SD & Difference $^{*}(95 \% \mathrm{Cl})$ & $P$ value \\
\hline \multicolumn{9}{|c|}{ Mini-Mental State Examination (MMSE) score } \\
\hline Initial & 21 & 22.6 & 5.6 & 25 & 23.4 & 4.5 & 0.00 & \\
\hline 0-3 months & 11 & 24.2 & 5.3 & 14 & 25.5 & 3.6 & $0.48(-2.51$ to 2.51$)$ & 0.65 \\
\hline 3-9 months & 21 & 22.2 & 6.1 & 24 & 26.4 & 3.8 & 3.35 (0.98 to 5.72 ) & 0.006 \\
\hline 9-16 months & 16 & 22.3 & 5.9 & 15 & 27.8 & 3.2 & 4.75 (0.93 to 8.57$)$ & 0.015 \\
\hline \multicolumn{9}{|c|}{ Tinetti† Balance and Gait score } \\
\hline Initial & 21 & 13.9 & 7.1 & 25 & 14.5 & 7.5 & 0.00 & \\
\hline $0-3$ months & 11 & 14.6 & 7.9 & 14 & 21.7 & 3.7 & 6.54 (1.66 to 11.4$)$ & 0.009 \\
\hline 3-9 months & 21 & 14.1 & 8.3 & 24 & 21.8 & 4.5 & 7.09 (3.43 to 10.8$)$ & $<0.001$ \\
\hline 9-16 months & 16 & 15.4 & 7.6 & 15 & 19.5 & 9.5 & $3.53(-0.42$ to 7.47$)$ & 0.080 \\
\hline \multicolumn{9}{|c|}{ Tinetti† Balance score } \\
\hline Initial & 21 & 8.0 & 4.0 & 25 & 8.4 & 4.2 & 0.00 & \\
\hline 0-3 months & 11 & 8.7 & 4.8 & 14 & 12.8 & 2.4 & 3.62 (0.78 to 6.46$)$ & 0.013 \\
\hline 3-9 months & 21 & 7.8 & 4.7 & 24 & 12.9 & 2.5 & 4.69 (2.54 to 6.84$)$ & $<0.001$ \\
\hline 9-16 months & 16 & 8.2 & 4.4 & 15 & 11.4 & 5.6 & 5.35 (0.51 to 5.12$)$ & 0.017 \\
\hline \multicolumn{9}{|c|}{ Tinetti† Gait score } \\
\hline Initial & 21 & 5.9 & 3.2 & 25 & 6.1 & 3.4 & 0.00 & \\
\hline $0-3$ months & 11 & 5.9 & 3.8 & 14 & 9.0 & 1.5 & 2.87 (0.62 to 5.12$)$ & 0.013 \\
\hline 3-9 months & 21 & 6.3 & 3.7 & 24 & 9.0 & 2.1 & 2.42 (0.73 to 4.10$)$ & 0.005 \\
\hline $9-16$ months & 16 & 7.2 & 3.4 & 15 & 8.2 & 3.9 & $0.71(-1.11$ to 2.52$)$ & 0.45 \\
\hline
\end{tabular}

*Mean difference in scores between shunted and non-shunted participants, estimated using repeated measures mixed effects linear regression adjusted for initial MMSE score and Tinetti scores (respectively), with the time from initial assessment of the follow-up observations being treated as a random variable.

†Tinetti Balance and Gait scores have 17-point (0-16) and 13-point (0-12) scales, respectively, with the combined scale being an addition of the two scales.

when including clinical and radiological variables. These index test diagnostic criteria are consistent with, although not precisely coincident with, the 'probable INPH' category as suggested by the International Guidelines criteria for INPH, and they are more precise than the "possible INPH' category, ${ }^{3}$ which would include a number of patients not diagnosed with INPH in this study. Ventriculomegaly including larger posterior horns is prevalent in INPH, possibly corresponding to smaller callosal angle measured at level of posterior commissure that has been used for INPH evaluation, ${ }^{24}$ although posterior cerebral atrophy from neurodegenerative disease such as Alzheimer's disease may confound this significance. DESH is a set imaging findings of ventriculomegaly, ${ }^{7}$ high convexity and medial subarachnoid space effacement, and enlargement of sylvian fissures that has been used for selecting patients in the Study of Idiopathic Normal Pressure Hydrocephalus on Neurological Improvement (SINPHONI and SINPHONI-2) trials,${ }^{25} 26$ although it should be noted that some INPH shunt-responsive patients do not have DESH, and not all patients who had DESH respond to shunting, so DESH is not necessary nor sufficient for the diagnosis of INPH. However, we did not have these measured at the time of examination to enable us to incorporate them in triage index test. We hope we can look at these in future studies.

There is high degree of overlap between individual symptoms and signs of INPH and non-INPH, which might lead to misdiagnoses of INPH as other neurodegenerative disorders including $\mathrm{AD}$, Parkinson's disease and vascular dementia. In addition, patients with INPH may have comorbidities affecting cognition such as $\mathrm{AD}$ and cerebrovascular disease. ${ }^{27}{ }^{28}$ Ventriculomegaly, the radiological hallmark of INPH, was present in about $18 \%$ of non-INPH study participants and was associated with cerebral atrophy. This makes it difficult to determine if the ventricular enlargement in the elderly results from hydrocephalus or cerebral atrophy. An Evans ratio threshold for ventriculomegaly relevant to the diagnosis of INPH has not been validated previously.

A CDT that creates an individual Evans ratio threshold for each patient, based on their MWPH of lateral 
Table 6 Change in subjective CIBIC plus scores over the 12 months following initial assessment in shunted and non-shunted participants

\begin{tabular}{|c|c|c|c|c|c|c|c|c|}
\hline & \multicolumn{4}{|c|}{ Non-shunted } & \multicolumn{2}{|c|}{ Shunted } & \multicolumn{2}{|c|}{$\begin{array}{l}\text { Comparison: shunted versus } \\
\text { non-shunted patients }\end{array}$} \\
\hline & $\mathbf{N}$ & Mean & SD & $\mathbf{N}$ & Mean & SD & Difference * (95\% Cl) & $P$ value \\
\hline \multicolumn{9}{|l|}{ Global $\dagger$} \\
\hline Initial & 5 & -0.5 & 1.2 & 13 & -1.4 & 0.9 & 0.00 & \\
\hline 0-3 months $\ddagger$ & 1 & -0.9 & 0.0 & 18 & 2.2 & 1.0 & 4.00 (1.3 to 6.7$)$ & 0.004 \\
\hline 3-9 months & 21 & -0.7 & 0.9 & 20 & 2.2 & 1.1 & 3.73 (2.67 to 4.79 ) & $<0.001$ \\
\hline $9-16$ months & 13 & -0.8 & 1.3 & 18 & 2.0 & 1.1 & 3.70 (2.38 to 5.03 ) & $<0.001$ \\
\hline \multicolumn{9}{|l|}{ Dementia } \\
\hline Initial & 5 & -0.5 & 1.3 & 13 & -1.2 & 0.8 & 0.00 & \\
\hline 0-3 months $\ddagger$ & 1 & 0.4 & 0.0 & 18 & 1.7 & 0.9 & 2.05 (-0.3 to 4.4$)$ & 0.09 \\
\hline 3-9 months & 21 & -0.9 & 0.8 & 20 & 1.8 & 1.2 & 3.42 (2.51 to 4.33$)$ & $<0.001$ \\
\hline 9-16 months & 13 & -0.7 & 1.4 & 18 & 2.0 & 1.2 & 3.41 (2.22 to 4.59$)$ & $<0.001$ \\
\hline \multicolumn{9}{|l|}{ Balance/gait } \\
\hline Initial & 5 & -0.9 & 1.1 & 13 & -0.9 & 0.8 & 0.00 & \\
\hline 0-3 months $\ddagger$ & 1 & -0.2 & 0.0 & 18 & 2.2 & 0.9 & $2.34(-0.3$ to 5.0$)$ & 0.09 \\
\hline 3-9 months & 21 & -1.1 & 1.0 & 20 & 2.3 & 1.0 & 3.43 (2.35 to 4.50$)$ & $<0.001$ \\
\hline 9-16 months & 13 & -1.3 & 1.2 & 18 & 1.8 & 1.4 & 3.07 (1.65 to 4.49$)$ & $<0.001$ \\
\hline \multicolumn{9}{|l|}{ Urinary } \\
\hline Initial & 5 & -0.4 & 1.2 & 13 & -0.5 & 0.7 & 0.00 & \\
\hline $0-3$ months & 1 & -0.4 & 0.0 & 18 & 1.7 & 1.6 & $2.16(-1.2$ to 5.5$)$ & 0.21 \\
\hline 3-9 months & 21 & -0.6 & 0.9 & 20 & 1.5 & 1.2 & 2.11 (0.90 to 3.32) & 0.001 \\
\hline $9-16$ months & 13 & -0.5 & 1.0 & 17 & 1.7 & 1.4 & 2.24 (0.87 to 3.62 ) & 0.001 \\
\hline
\end{tabular}

*Mean difference in scores between shunted and non-shunted participants, estimated using repeated measures mixed effects linear regression adjusted for initial CIBIC-plus scores, with the time from initial assessment of the follow-up observations being treated as a random variable.

†CIBIC-plus scores have a 7-point scale format: very much worsening, moderately worsening, minimally worsening, unchanged, minimally improved, moderately improved, very much improved.

CIBIC-plus, Clinician's Interview Based Impression of Change Plus Caregiver Interview.

ventricles, might be a better predictor of the diagnosis of INPH. However, this is a preliminary finding and requires further refinement of the diagnostic definition using CT brain imaging. Moreover, intracranial measurements can vary depending on the location measured and axial slice angle. For CT scans and other imaging techniques conducted in specialist INPH assessment centres, the head position and slices angles need to be standardised. Additionally, day-to-day variations in patient positioning as well as variations in scanning protocol from institution to institution need to be taken into account.

\section{Outcome of shunt surgery}

Studies on shunting for INPH revealed variable outcomes. ${ }^{218} 2930$ This may be due to difference in patient selection criteria and postsurgical outcome measures. An earlier meta-analysis of 44 studies reported a low rate of improvement following shunting, with approximately $59 \%$ experiencing short-term and 29\% experiencing prolonged improvement. ${ }^{2}$ A recent review of 64 studies reported an average improvement of $71 \%$ following shunting, ${ }^{29}$ with improvement rates reaching $90 \%$ using of supplementary tests. ${ }^{30}$

It has been suggested that cognitive function is the least likely to improve following shunting leading to suggestion that INPH in the elderly should be considered a treatable gait disorder rather than treatable dementia. ${ }^{18}$ Furthermore, the presences of severe dementia, marked cerebral atrophy and extensive white matter ischaemia, have been used to exclude patients for shunt surgery. ${ }^{231} 32$ However, a recent systematic review of 23 studies suggested an improvement of global cognitive function, verbal learning and memory and psychomotor speed following shunting. ${ }^{33}$ In our study, the majority of patients with INPH showed improvement of at least $25 \%$ following shunting in either MMSE or balance/gait scores, and $56 \%$ improved by at least $50 \%$ in both in the first year. There was no association between the severity of initial symptoms and response to shunting, and the more pronounced symptoms were not associated with greater improvement. 


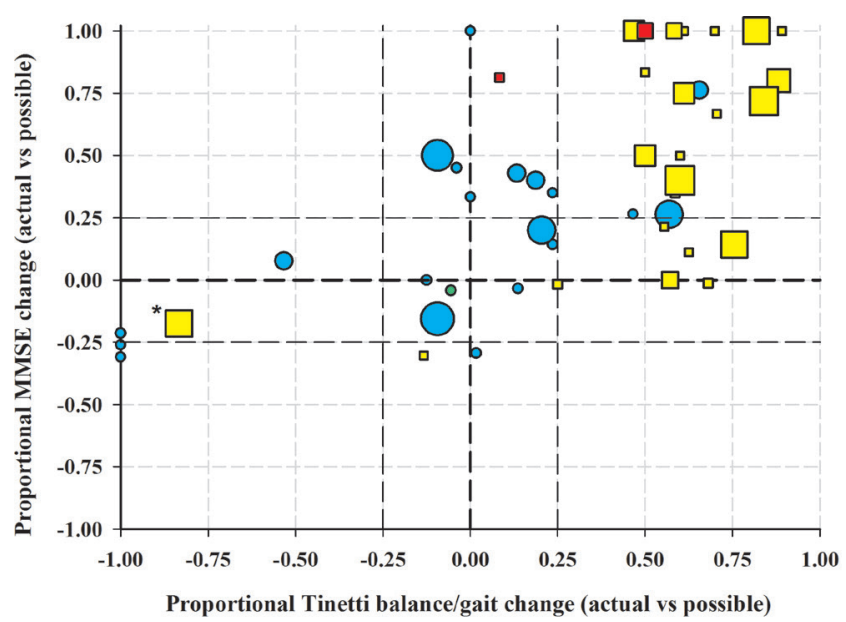

- Unshunted: International Guidelines and Launceston both positive $\quad(\mathrm{N}=20)$ Unshunted: International Guidelines negative; Launceston positive ( $\mathrm{N}=1)$ Shunted: International Guidelines negative; Launceston positive Shunted: International Guidelines and I aunceston both positive

Marker sizes are inversely proportional to the patient's diagnostic risk score: Larger markers indicate patients with lower diagnostic index scores

3 participants with apparent $100 \%$ reduction in Tinetti scores could not complete the tests

* Woman had stroke 3 weeks after shunt causing visual disturbance, resulting in reduced balance, with declines in balance/gait test and MMSE scores

Figure 4 Association between proportional change in MMSE and Tinetti combined balance and gait scores in the shunted and non-shunted participants: mean change at all follow-up visits between 3 and 16 months (nominal 6 month and 12 month clinic visits). MMSE, Mini-Mental State Examination.

\section{Strengths and limitations of the study}

The study of the incidence of INPH among patients with memory problems is epidemiologically robust, being prospective within a defined population and a wellestablished clinical service. The detailed clinical and radiological assessments and diagnoses are likely to have identified patients at early stages of the disease, while minimising ascertainment bias. Moreover, all presurgical and postsurgical assessments were performed independent of the neurosurgeons, which reduced any selection or treatment-effect bias. The incidence of INPH among the Launceston memory clinic population has remained consistent over the last decade, ${ }^{10}$ maintained by the continuous awareness of the importance of diagnosing INPH.

There were limitations specific to this study. First, the lack of data on asymptomatic INPH or patients without memory problems. This is more likely to underestimate the true incidence of INPH. Second, there was the potential for bias due to missing data. The number of cases with missing data was relatively small $(7.8 \%)$, and the method of substitution for that missing data was pessimistic, assuming random distribution of data when it was more likely that the missing data occurred with non-recording of negative findings. The more optimistic assumption predicted a specificity of $67.3 \%$ instead of $64.7 \%$ for the missing-at-random assumption. Third, the triage index test scores need to be externally validated in new populations to validate the scores with confidence. Fourth, the study was not a randomised, blinded or placebo-controlled clinical trial due to ethical concerns that delaying accepted treatment in the control group allocation could lead to deterioration of the condition while waiting for trial follow-up. ${ }^{34}$ However, a recent open-labelled randomised trial of lumboperitoneal shunt surgery showed that shunt surgery improved functional status and symptoms for patients with INPH. ${ }^{26}$

\section{Conclusion and implications}

This study has developed a simple assessment tool to predict the diagnosis of INPH which might be used by clinicians in non-specialised settings. The findings might improve timely and accurate recognition of the disorder, increase the awareness of clinicians to the higher incidence of INPH among patients with memory problems and dementia, and that V-P shunting may help to improve cognitive, balance, gait and urinary functioning and overall quality of life. The diagnostic criteria and the incidence of INPH require further evaluation in different populations.

\section{Author affiliations}

${ }^{1}$ Department of Medicine, Launceston General Hospital, and Launceston Clinical School, University of Tasmania, Launceston, Tasmania, Australia

${ }^{2}$ Department of Medicine, Launceston General Hospital, Dementia Research Centre, Launceston, Tasmania, Australia

${ }^{3}$ College of Health and Medicine, University of Tasmania, Launceston, Tasmania, Australia

Acknowledgements We thank all participants and their carers for their time and commitment to our study, Anthea Vreugdenhil, School of Social Sciences, University of Tasmania, for helping in designing the study, and the Neurosurgeons at the Royal Hobart Hospital, Hobart, Tasmania (A Hunn, A Erasmus, A Dubey, J Peters-Wilke, A Mujic) for supporting the study and performing V-P shunt surgery.

Contributors GR and IR conceived, designed the study and drafted the manuscript; MW collected the data; IR undertook the statistical analysis, all authors had full access to all the data, including statistical reports and tables; all authors critically revised the manuscript for important intellectual content. GR is guarantor.

Funding Funding was granted by the Clifford Craig Foundation, Launceston, Australia, a non-profit local organisation.

Disclaimer The opinion, results and conclusions reported in the paper are independent of the funding source.

Competing interests None declared.

Patient consent for publication Not required.

Ethics approval The study was approved by the Tasmanian Health and Medical Human Research Ethics Committee and informed consent was provided by all participants or their guardians.

Provenance and peer review Not commissioned; externally peer reviewed.

Data availability statement All data relevant to the study are included in the article or uploaded as online supplementary information.

Open access This is an open access article distributed in accordance with the Creative Commons Attribution Non Commercial (CC BY-NC 4.0) license, which permits others to distribute, remix, adapt, build upon this work non-commercially, and license their derivative works on different terms, provided the original work is properly cited, appropriate credit is given, any changes made indicated, and the use is non-commercial. See: http://creativecommons.org/licenses/by-nc/4.0/.

\section{REFERENCES}

1 Hakim S, Adams RD. The special clinical problem of symptomatic hydrocephalus with normal cerebrospinal fluid pressure. 
Observations on cerebrospinal fluid hydrodynamics. J Neurol Sci 1965;2:307-27.

2 Hebb AO, Cusimano MD. Idiopathic normal pressure hydrocephalus: a systematic review of diagnosis and outcome. Neurosurgery 2001;49:1166-84. discussion 1184-6.

3 Relkin N, Marmarou A, Klinge P, et al. Diagnosing idiopathic normalpressure hydrocephalus. Neurosurgery 2005;57(3 Suppl):S4-16. discussion ii-v.

4 Klassen BT, Ahlskog JE. Normal pressure hydrocephalus: how often does the diagnosis hold water? Neurology 2011;77:1119-25.

5 Brean A, Eide PK. Prevalence of probable idiopathic normal pressure hydrocephalus in a Norwegian population. Acta Neurol Scand 2008;118:48-53.

6 Jaraj D, Rabiei K, Marlow T, et al. Prevalence of idiopathic normalpressure hydrocephalus. Neurology 2014;82:1449-54.

7 Mori E, Ishikawa M, Kato T, et al. Guidelines for management of idiopathic normal pressure hydrocephalus: second edition. Neurol Med Chir 2012;52:775-809.

8 Clarfield AM. The decreasing prevalence of reversible dementias: an updated meta-analysis. Arch Intern Med 2003;163:2219-29.

9 Knopman DS, Petersen RC, Cha RH, et al. Incidence and causes of nondegenerative nonvascular dementia: a population-based study. Arch Neurol 2006;63:218-21.

10 Razay G, Vreugdenhil A, Liddell J. A prospective study of ventriculoperitoneal shunting for idiopathic normal pressure hydrocephalus. $J$ Clin Neurosci 2009;16:1180-3.

11 Folstein MF, Folstein SE, McHugh PR. "Mini-mental state". A practical method for grading the cognitive state of patients for the clinician. J Psychiatr Res 1975;12:189-98.

12 Sorbi S, Hort J, Erkinjuntti T, et al. EFNS-ENS guidelines on the diagnosis and management of disorders associated with dementia. Eur J Neurol 2012;19:1159-79.

13 Petersen RC, Smith GE, Waring SC, et al. Mild cognitive impairment: clinical characterization and outcome. Arch Neurol 1999:56:303-8.

14 Knopman DS, Knapp MJ, Gracon SI, et al. The clinician InterviewBased impression (CIBI): a clinician's global change rating scale in Alzheimer's disease. Neurology 1994;44:2315-21.

15 Tinetti ME. Performance-oriented assessment of mobility problems in elderly patients. J Am Geriatr Soc 1986;34:119-26.

16 Mataró M, Poca MA, Sahuquillo J, et al. Cognitive changes after cerebrospinal fluid shunting in young adults with spina bifida and assumed arrested hydrocephalus. J Neurol Neurosurg Psychiatry 2000;68:615-21.

17 Frisoni GB, Scheltens Ph, Galluzzi S, et al. Neuroimaging tools to rate regional atrophy, subcortical cerebrovascular disease, and regional cerebral blood flow and metabolism: consensus paper of the EADC. J Neurol Neurosurg Psychiatry 2003;74:1371-81.

18 Krauss JK, Halve B. Normal pressure hydrocephalus: survey on contemporary diagnostic algorithms and therapeutic decisionmaking in clinical practice. Acta Neurochir 2004;146:379-88. discussion 388.

19 Tisell M, Höglund M, Wikkelsø C. National and regional incidence of surgery for adult hydrocephalus in Sweden. Acta Neurol Scand 2005;112:72-5
20 Martín-Láez R, Caballero-Arzapalo H, López-Menéndez Luis Ángel, et al. Epidemiology of idiopathic normal pressure hydrocephalus: a systematic review of the literature. World Neurosurg 2015;84:2002-9.

21 Iseki C, Kawanami T, Nagasawa H, et al. Asymptomatic ventriculomegaly with features of idiopathic normal pressure hydrocephalus on MRI (AVIM) in the elderly: a prospective study in a Japanese population. J Neurol Sci 2009;277:54-7.

22 Marmarou A, Young HF, Aygok GA. Estimated incidence of normal pressure hydrocephalus and shunt outcome in patients residing in assisted-living and extended-care facilities. Neurosurg Focus 2007;22:1-8.

23 Wikkelsø C, Hellström P, Klinge PM, et al. The European iNPH multicentre study on the predictive values of resistance to CSF outflow and the CSF TAP test in patients with idiopathic normal pressure hydrocephalus. J Neurol Neurosurg Psychiatry 2013;84:562-8.

24 Ishii K, Kanda T, Harada A, et al. Clinical impact of the callosal angle in the diagnosis of idiopathic normal pressure hydrocephalus. Eur Radiol 2008;18:2678-83.

25 Hashimoto M, Ishikawa M, Mori E, et al. Diagnosis of idiopathic normal pressure hydrocephalus is supported by MRI-based scheme: a prospective cohort study. Cerebrospinal Fluid Res 2010;7:18.

26 Kazui H, Miyajima M, Mori E, et al. Lumboperitoneal shunt surgery for idiopathic normal pressure hydrocephalus (SINPHONI-2): an openlabel randomised trial. Lancet Neurol 2015;14:585-94.

27 Malm J, Graff-Radford NR, Ishikawa M, et al. Influence of comorbidities in idiopathic normal pressure hydrocephalus - research and clinical care. A report of the ISHCSF task force on comorbidities in INPH. Fluids Barriers CNS 2013;10:22.

28 Bech-Azeddine R, Høgh P, Juhler M, et al. Idiopathic normalpressure hydrocephalus: clinical comorbidity correlated with cerebral biopsy findings and outcome of cerebrospinal fluid shunting. $J$ Neurol Neurosurg Psychiatry 2007;78:157-61.

29 Toma AK, Papadopoulos MC, Stapleton S, et al. Systematic review of the outcome of shunt surgery in idiopathic normal-pressure hydrocephalus. Acta Neurochir 2013;155:1977-80.

30 Eide PK, Sorteberg W. Diagnostic intracranial pressure monitoring and surgical management in idiopathic normal pressure hydrocephalus: a 6-year review of 214 patients. Neurosurgery 2010;66:80-91.

31 Stolze H, Kuhtz-Buschbeck JP, Drücke H, et al. Comparative analysis of the gait disorder of normal pressure hydrocephalus and Parkinson's disease. J Neurol Neurosurg Psychiatry 2001;70:289-97.

32 Vanneste JA. Diagnosis and management of normal-pressure hydrocephalus. J Neurol 2000;247:5-14.

33 Peterson KA, Savulich G, Jackson D, et al. The effect of shunt surgery on neuropsychological performance in normal pressure hydrocephalus: a systematic review and meta-analysis. J Neurol 2016;263:1669-77.

34 Andrén K, Wikkelsø C, Tisell M, et al. Natural course of idiopathic normal pressure hydrocephalus. J Neurol Neurosurg Psychiatry 2014;85:806-10. 